\title{
Lack of relationship between masticatory performance and nutritional status in adults with natural dentition
}

\author{
Elan Ignacio Flores-Orozco a , Gaby Esthela Tiznado-Orozco a , Olga Dionicia Osuna-González ${ }^{\mathrm{a}}$, \\ Claudia Lucero Amaro-Navarrete ${ }^{\mathrm{a}}$, Bernat Rovira-Lastra ${ }^{\mathrm{b}, \mathrm{c}}$, Jordi Martinez-Gomis ${ }^{\mathrm{b}, \mathrm{c}, *}$ \\ ${ }^{a}$ Department of Prosthodontics, Faculty of Dentistry, Autonomous University of Nayarit, Mexico \\ ${ }^{\mathrm{b}}$ Department of Prosthodontics, Faculty of Dentistry, University of Barcelona, Spain \\ c Oral Health and Masticatory System Group (Bellvitge Biomedical Research Institute) IDIBELL, L'Hospitalet de Llobregat, Barcelona, Spain
}

\section{A R T I C L E I N F O}

\section{Article history:}

Received 3 January 2016

Received in revised form 15 July 2016

Accepted 27 July 2016

Available online $\mathrm{xxx}$

Keywords:

Masticatory performance

Anthropometric measurement

Obesity

Chewing rate

Preferred chewing side

\section{A B S T R A C T}

\section{Objective}

This study assessed the relation among several aspects of the masticatory function and the nutritional status in adults with natural dentition.

Design

One hundred adults with natural dentition participated in this cross-sectional study. They performed one free-style masticatory test consisting of five trials of 20 silicon-chewing cycles. The preferred chewing side was determined by calculating the asymmetry index. Masticatory performance was determined by sieving the silicon particles, and the cycle duration was also recorded. Weight, body water percentage, body fat mass, muscle mass and osseous mass were measured using a portable digital weighing machine. Body mass index (BMI), waist-hip ratio, skinfold thickness and the upper-arm composition were determined. The relation between masticatory function and a nutritional variable were tested using Pearson or Spearman rank correlation coefficients or using analysis of variance or the Kruskal-Wallis H-test and the Mann-Whitney $U$ test, as appropriate.

Results

Whereas body fat percentages for women were significantly higher than for men, the body mass index was higher in men than in women. Participants who were underweight chewed more asymmetrically and more slowly than normal weight or obese participants. A negative correlation was observed between body fat percentage and masticatory laterality. No relation between masticatory performance and any nutritional status indicator was detected.

Conclusion

Being underweight and having a low body fat percentage seem to be related to a masticatory lateral asymmetry and to a large cycle duration in young adults with natural dentition. Masticatory performance does not seem to be related to nutritional status.

(C) 2016 Published by Elsevier Ltd.

\section{Introduction}

Nutrition is a general term for referring to the process of obtaining nutrients in food for health and growth. There are complex interactions between nutrition and oral and general health (Walls \& Steele, 2004). The nutritional status is the state of the body in relation to the consumption and utilization of nutrients. Being overweight or obese is an important risk factor for morbidity from chronic diseases such as musculoskeletal disorders, cancers, diabetes and cardiovascular diseases. The body mass index (BMI) is normally used to assess being overweight and being obese, though percent body fat, waist-hip ratio and other anthropometric measurements are used as indicators of obesity (Panel, 1998). Several factors are normally involved in the

\footnotetext{
* Corresponding author at: Campus de Bellvitge, Universitat de Barcelona, C/Feixa llarga s/n, 08907 L'Hospitalet de Llobregat, Barcelona, Catalonia, Spain.

Email address: jmartinezgomis@gmail.com, jmartinezgomis@ub.edu (J. Martinez-Gomis)
}

generation of obesity, including genetic, physiological, metabolical, cultural, behavioral and social factors (Walls \& Steele, 2004). Mastication is the first stage of digestion and is the process in which food is fragmented into small particles that are ready to be swallowed (Van der Bilt, 2011).

Oral function includes a number of phenomena such as masticatory performance, masticatory laterality or chewing rate. Masticatory performance can be determined by quantifying the degree of fragmentation of a test food after a fixed number of chewing cycles (Lujan-Climent et al., 2008; Van der Bilt \& Fontijn-Tekamp, 2004). Dental silicone is considered the more appropriate test food (Edlund \& Lamm, 1980), and silicon pieces placed in a latex bag have been demonstrated to be a reliable method for assessing masticatory function in dentate young adults with natural dentition (Rovira-Lastra, Flores-Orozco, Salsench, Peraire, \& Martinez-Gomis, 2014). It is well known that the majority of people chew more on one particular side, i.e., they have a masticatory laterality (Diernberger, Bernhardt, Schwahn, \& Kordass, 2008; Martinez-Gomis et al., 2009; Rovira- 
Lastra, Flores-Orozco, Ayuso-Montero, Peraire, \& Martinez-Gomis, 2016). Although bilateral chewers were observed to chew more efficiently than unilateral chewers (Farias Gomes, Custodio, Moura Jufer, Del Bel Cury, \& Rodrigues Garcia, 2010; Rovira-Lastra et al., 2014), no direct relation has been demonstrated (Rovira-Lastra et al., 2014). Chewing rate, defined as the frequency of chewing cycles per unit of time, seems to be remarkably stable within individuals, even across different days and foods (White et al., 2015). It has been found that eating slowly is associated with lower body mass index in middle-aged women (Leong, Madden, Gray, Waters, \& Horwath, 2011).

Poor masticatory performance has been associated with both overweight and being underweight in children and with a lower mid-upper-arm circumference in the elderly (Okada, Enoki, Izawa, Iguchi, \& Kuzuya, 2010; Tureli, Barbosa, \& Gavião, 2010). However, apparently contradictory results have reported the relation between masticatory function and nutritional status in adults with natural dentition. Whereas the number of chewing cycles before swallowing was found to be negatively associated with BMI in a recent study, a positive association between BMI and the number of masticatory cycles was reported in another study (Sánchez-Ayala, Campanha, \& Garcia, 2013; Zhu, \& Hollis, 2015).

The main objective of this study was to determine the relationship between masticatory performance and the nutritional status in young adults with natural dentition. This study also assessed the relationship between masticatory laterality or chewing rate and the nutritional status in the same population. The null hypothesis was that masticatory performance is not associated with nutritional status.

\section{Materials and methods}

\subsection{Subjects}

Young adults with natural dentition among volunteer students and staff at the Faculty of Dentistry, Autonomous University of Nayarit, Tepic, Mexico participated in this cross-sectional study. Individuals with fewer than 24 natural teeth, without any edentulous space, those undergoing active orthodontic treatment and those suffering orofacial pain were excluded. No participant had severe malocclusion or temporomandibular disorders that could affect mandibular movement. Of the 103 participants who were invited to enroll in this study, three were excluded because of the lack of data. Thus, 100 participants were included, with a mean age of 21.9 years, and $53 \%$ of the sample were women. All participants provided written informed consent. The study was approved by the Ethics Committee of the Faculty of Medicine, Autonomous University of Nayarit, and all experiments were conducted in accordance with the principles of the Helsinki Declaration (World Medical Association, 2013).

\subsection{Masticatory assays}

The subjects were asked to chew naturally without imposing any side of the mouth for mastication and count their chewing cycles. A test medium known as "bagged silicone" was used for 20 cycles to comminute the pieces (Flores-Orozco, Rovira-Lastra, Willaert, Peraire, \& Martinez-Gomis, 2016). Optosil tablets (5 mm thick, 20-mm diameter) were produced as described by Albert, Buschang, \& Throckmorton (2003), and cut into quarters, and three quarters of a tablet was placed in a latex bag, which was sealed with cyanoacrylate adhesive (Rovira-Lastra et al., 2014). The masticatory assay was repeated four more times, and the particles from the five assays were collected to evaluate the masticatory performance. Particles from five trials were dried for $24 \mathrm{~h}$ and passed through a series of eight sieves $(0.25,0.425,0.85,2,2.8,3.35,4$, and $5.6 \mathrm{~mm})$ while being shaken for 2 min (-Climent et al., 2008). After cumulative weight distribution of the sieves contents had been determined, median particle size was calculated for each subject using the Rosin-Rammler equation $\left[\mathrm{Qw}(\mathrm{X})=1-2 \mathrm{E}-\left(\mathrm{X} / \mathrm{X}_{50}\right)^{\mathrm{b}}\right]$, where $\mathrm{Qw}(\mathrm{X})$ is the fraction of particles by weight with a diameter smaller than $X$. The median particle size $\left(\mathrm{X}_{50}\right)$ is the size of a theoretical sieve through which $50 \%$ of the weight can pass, and $\mathrm{b}$ describes the broadness of the particle distribution (Olthoff, Van Der Bilt, Bosman, \& Kleizen, 1984). Therefore, the lower the median particle size value, the better the masticatory performance.

To determine the preferred chewing side, one operator observed from the frontal plane on the side towards which the jaw moved while closing for each masticatory cycle. Therefore, each cycle was classified as right-, left- or no-side and recorded by means of two hand counters (Flores-Orozco, 2014; Flores-Orozco, Rovira-Lastra, Peraire, Salsench, \& Martinez-Gomis, 2016). Questionable strokes were considered as no-side cycle. All masticatory cycles were considered to calculate the asymmetry index $(\mathrm{AI})$ as follows: $\mathrm{AI}=$ (number of right strokes - number of left strokes)/(number of right strokes + number of left strokes) (Mizumori, Tsubakimoto, Iwasaki, \& Nakamura, 2003). Masticatory laterality was considered as the absolute AI value. Therefore, values close to zero meant masticatory symmetry and values close to 1 meant masticatory asymmetry.

The total duration of the five masticatory assays was used to calculate the duration of an average chewing cycle (Salsench et al., 2005).

\subsection{Anthropometric measurements}

All subjects were fasting and with bare feet and comfortable clothing (underwear or sportswear) worn during measurements. Weight, height, body circumferences and skinfold thickness were recorded three times using standard procedures (Gibson, 2005). Weight, body water percentage, body fat mass, muscle mass and osseous mass were measured using a portable digital weighing machine (TANITA BC-553, Tanita Corporation of America Inc., Arlington Heights, Il USA). Height was measured using a wall mounted stadiometer (SECA 206, length of $2200 \mathrm{~mm}$, accuracy $1 \mathrm{~mm}$ ). Waist circumference was measured with a flexible tape MASS ${ }^{\circledR}$ (Accuracy $1 \mathrm{~mm}$ ) in the time gap between the end of exhalation and the beginning of the inspiration of normal breathing, midway between lower edge of the tenth rib and the iliac crest. Mid-upper arm circumference (MAC) was taken at the point midway between the acromion and the radiale of the upper arm using a flexible tape on the left side. Skinfold thickness (biceps "BFS", triceps "TFS", subscapular "SSF", suprailiaco "ISF") were recorded following the criteria of Durnin and Womersley (1974) using a Slim Guide ${ }^{\circledR}$ skinfold caliper calibrated to exert a constant pressure of $10 \mathrm{gm} / \mathrm{mm}^{2}$. The anthropometric measurements were collected by a previously calibrated single observer.

\subsection{Assessment of body composition}

Body mass index (BMI) was calculated as body weight divided by height squared. Waist-hip ratio (WWR) using a tape was determined as waist measurement divided by hip measurement. The upper arm composition was assessed based on anthropometric measurements of MAC and TSF using standard equations (Martín Moreno, Gómez Gandoy, Antoranz González, \& Gómez de la Cámara, 2003):

a) Upper arm muscle perimeter "AMP" $=\mathrm{MAC}-(3.14 \times \mathrm{TFS})$

b) Upper arm muscle area "AMA" $=(\mathrm{AMP})^{2} /(4 \times 3.14)$

c) Upper arm fat area "AFA" $=$ TFS* $(\mathrm{MAC} / 2)-\left(\left(3.14 \times \mathrm{TFS}^{*}\right)^{2} / 4\right)$ (*.- Measure in centimeters) 
d) Upper arm adipose muscle index "AAMI" = AFA/AMA

The body composition was assessed based on anthropometric measurements of skinfold thickness, weight and height. Body fat percentage was calculated using of body density " $D$ " that is obtained through these equations (Moreno, Gomez Gandoy, \& Gonzales, 2001):

a) $\%$ Body Fat $($ Siri $)=((4.95 /$ density $)-4.5) \times 100$

b) $\%$ Body Fat $($ Brozeck $)=((4.57 /$ density $)-4.142) \times 100$

c) $\%$ Corporal Body Fat "CBF" $=((\%$ Body Fat Siri $)+(\%$ Body Fat Brozeck)) $/ 2$

Body fat total, free fat mass and muscle mass were obtained through these mathematic formulas (Suverza \& Haua, 2010):

- Body Fat Total $(\mathrm{kg})$ "BFT" $=($ Weight $\mathrm{kg} \times \mathrm{CBF}) / 100$

- Fat Free Body mass (kg) "FFB" = Weight kg - BFT

- Muscle Mass Total (kg) "MMT" = $($ Height $\mathrm{cm})$ $(0.0264+(0.0029 \times$ AMA $)$

\subsection{Biochemical assessment}

Blood samples were collected after fasting. Serum total protein, albumin and total cholesterol levels were determined using automated analyzers.

\subsection{Data analysis}

A new qualitative variable called Obesity with four categories was created from BMI values, with the categories being Underweight if $\mathrm{BMI}<18.5 \mathrm{~kg} / \mathrm{m}^{2}$; Normal weight if $18.5-24.9 \mathrm{~kg} / \mathrm{m}^{2}$; Overweight if $25.0-29.9 \mathrm{~kg} / \mathrm{m}^{2}$ and Obese if BMI $\geq 30 \mathrm{~kg} / \mathrm{m}^{2}$ (Panel, 1998). The normal distribution of the data was tested by means of a Kolmogorov-Smirnov test. Pearson or Spearman rank correlation coefficients were calculated to evaluate the bivariate correlation between the nutritional parameters and masticatory parameters (median particle size, laterality masticatory and chewing cycle duration). Comparisons between being underweight, normal, overweight and obese were performed using analysis of variance or the Kruskal-Wallis H-test and the Mann-Whitney $U$ test, as appropriate. Statistical analysis was performed using the SPSS program (IBM SPSS Statistics, version 22.0.0.0, Chicago, IL). P-values below 0.05 were considered significant.

\section{Results}

Data from nutritional and anthropometric indicators are shown in Table 1 . Whereas body fat percentage for women were significantly higher than for men, body mass index was higher in men than in women. However, there were no differences between the sexes in the masticatory performance, masticatory laterality or cycle duration.

No relation between masticatory performance and any nutritional status indicator was detected. Participants who were underweight chewed more asymmetrically $(\mathrm{P}<0.05$; Mann-Whitney $U$ test $)$ than normal weight participants (Table 2). Moreover, underweight subjects chewed more slowly compared with obese participants (Table 2). A negative correlation was observed between body fat percentage measured with Tanita and masticatory laterality (Spearman Rho $=-0.27$; $\mathrm{P}=0.006$ ), that is, the less body fat, the more asymmetrical was the mastication (Fig. 1). Upper arm adipose muscle index correlated positively with chewing cycle duration (Pearson $\mathrm{r}=0.26 ; \mathrm{P}=0.008$ ), that is, participants with a greater muscular mass chewed faster than those with smaller muscular mass (Fig. 2).
Table 1

Comparison by sex of variables related to nutritional status (anthropometric measurements and biochemical parameters) and masticatory function.

\begin{tabular}{|c|c|c|c|}
\hline & Men & Sex differences & Women \\
\hline & $(n=47)$ & $(P$-value $)$ & $(\mathrm{n}=53)$ \\
\hline $\begin{array}{l}\text { Age (Years) Median } \\
\text { Anthropometric measurements }\end{array}$ & 22.2 & $0.02 *$ & 21.3 \\
\hline TAN_Weight (kg) Mean (SD) & $77.6(15)$ & $<0.001$ & $60.6(12)$ \\
\hline TAN_Height (m) Mean (SD) & $1.75(0.07)$ & $<0.001$ & $1.63(0.05)$ \\
\hline TAN_Fat (\%) Mean (SD) & $19.7(7.1)$ & $<0.001$ & $27.2(8.3)$ \\
\hline TAN_Water (\%) Mean (SD) & $56.8(4.9)$ & $<0.001$ & $51.0(5.0)$ \\
\hline TAN_Muscle Mass (kg) Median & 57.7 & $<0.001^{*}$ & 40.2 \\
\hline TAN_Osseus body (kg) Median & 3.00 & $<0.001^{*}$ & 2.20 \\
\hline BMI $(\mathrm{kg} / \mathrm{m} 2)$ Mean (SD) & $25.4(4.2)$ & 0.002 & $22.8(4.1)$ \\
\hline Waist Hip Ratio Mean (SD) & $0.83(0.05)$ & $<0.001$ & $0.74(0.04)$ \\
\hline AMP (cm) Mean (SD) & $28.0(3.4)$ & $<0.001$ & $22.9(2.8)$ \\
\hline AMA (cm2) Mean (SD) & $63.5(16)$ & $<0.001$ & $42.3(11)$ \\
\hline AFA $(\mathrm{cm} 2)$ Median & 17.0 & $0.31^{*}$ & 18.1 \\
\hline AAMI Mean (SD) & $0.28(0.09)$ & $<0.001$ & $0.45(0.10)$ \\
\hline CBF (\%) Mean (SD) & $16.4(3.9)$ & $<0.001$ & $25.2(3.6)$ \\
\hline BFT $(\mathrm{Kg})$ Median & 12.2 & $0.009 *$ & 14.5 \\
\hline FFB $(\mathrm{kg})$ Median & 64.0 & $<0.001^{*}$ & 42.4 \\
\hline MMT (kg) Median & 34.7 & $<0.001^{*}$ & 23.2 \\
\hline \multicolumn{4}{|l|}{ Biochemical parameters } \\
\hline Cholesterol Median & 179 & 0.60 & 178 \\
\hline Albumin Median & 4.40 & $0.50 *$ & 4.10 \\
\hline Protein Mean (SD) & $6.94(0.65)$ & 0.10 & $7.15(0.64$ \\
\hline \multicolumn{4}{|l|}{ Masticatory function } \\
\hline Median Particle Size (mm) Median & 3.9 & $0.58^{*}$ & 3.9 \\
\hline Masticatory laterality Median & 0.22 & 0.14 & 0.20 \\
\hline Cycle duration (msec) Mean (SD) & $758(142)$ & 0.32 & $780(90)$ \\
\hline
\end{tabular}

TAN - Tanita, BMI - Body mass index, AMP - Upper arm muscle perimeter, AMA Upper arm muscle area, AFA - Upper arm fat area, AAMI - Upper arm adipose muscle index, CBF - Corporal body fat, BFT - Total body fat, FFB - Fat free body, MMT Muscle mass total. Analyzed by Student-T test or * Mann Whitney U Test. Applying Bonferroni Correction, $P<0.002$ was considered significant.

Table 2

Masticatory characteristics according to obesity group.

\begin{tabular}{lllll}
\hline & $\mathrm{N}$ & $\begin{array}{l}\text { Median Particle Size } \\
(\mathrm{mm})\end{array}$ & $\begin{array}{l}\text { Masticatory } \\
\text { laterality }\end{array}$ & $\begin{array}{l}\text { Cycle Duration } \\
(\mathrm{msec})\end{array}$ \\
\hline & & Median & Median & Mean (SD) \\
\hline Underweight & 6 & $3.58^{\mathrm{a}}$ & $0.80^{\mathrm{b}}$ & $837(73)^{\mathrm{d}}$ \\
Normal & 58 & $3.86^{\mathrm{a}}$ & $0.21^{\mathrm{c}}$ & $779(117)^{\mathrm{d}, \mathrm{e}}$ \\
$\begin{array}{l}\text { weight } \\
\text { Overweight }\end{array}$ & 28 & $3.80^{\mathrm{a}}$ & $0.20^{\mathrm{b}, \mathrm{c}}$ & $753(120)^{\mathrm{d}, \mathrm{e}}$ \\
Obesity & 8 & $3.55^{\mathrm{a}}$ & $0.31^{\mathrm{b}, \mathrm{c}}$ & $722(117)^{\mathrm{e}}$ \\
Total & 100 & 3.79 & 0.21 & $770(117)$ \\
\hline
\end{tabular}

Different letters indicate significant differences between groups $(P<0.05)$ according to the Kruskal-Wallis H-test and the Mann-Whitney $U$ test for median particle size and masticatory laterality and ANOVA and the post hoc Duncan test for cycle duration.

\section{Discussion}

Although some aspects of masticatory function were associated with obesity in the present study, no significant associations between masticatory performance and obesity or body fat percentage was detected, therefore the null hypothesis was not rejected. It was reported that poor masticatory performance was associated with both overweight and being underweight in children (Tureli, Barbosa, \& Gavião, 2010), with an increased body fat in adults and with lower mid-upper arm circumference in the elderly (Sánchez-Ayala, Campanha, \& Garcia, 2013; Okada et al., 2010). However, these studies were performed in a more heterogeneous population including 


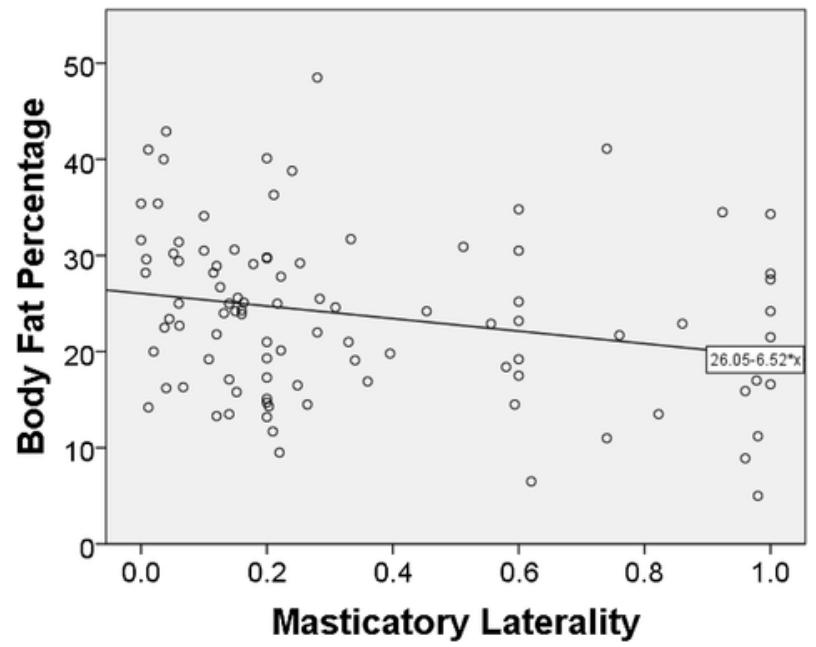

Fig. 1. Correlation between body fat percentage measured with Tanita and masticatory laterality.

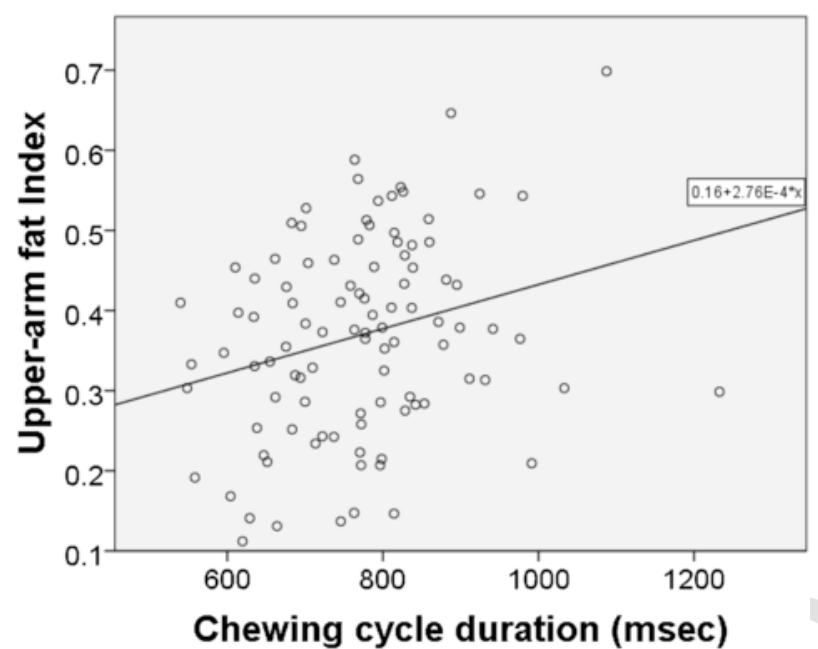

Fig. 2. Positive correlation between upper arm adipose muscle index and duration of chewing cycle.

children with mixed dentition (Tureli, Barbosa, \& Gavião, 2010), the elderly recruited from a geriatric clinic or a rehabilitation hospital (Okada et al., 2010), and adults with 0-14 occlusal pairs recruited from a university dental clinic (Sánchez-Ayala et al., 2013). Consequently, interindividual differences in masticatory performance in young people with natural dentition are not high enough to detect a relation between masticatory performance and being overweight or obese. Further studies are needed to clarify the influence of masticatory performance and obesity in an adult population.

Masticatory laterality and chewing rate were associated with obesity in a young adult population with natural dentition. Being underweight, expressed not only as low values of BMI but also as low values of body fat percentage, have an habitual side for chewing. It is known that harder foods require more effort than softer foods and probably people use the preferred chewing side for comminuting hard food (Mizumori, Tsubakimoto, Iwasaki, \& Nakamura, 2003). Eating food with fibrous consistency, such as raw vegetables, requires more chewing cycles on the preferred side, which is the more efficient side for breakdown of food and to allow swallowing (Paphangkorakit, Thothongkam, \& Supanont, 2006). Therefore, a lesser amount of calories is ingested in a greater amount of time. Consequently, the re- lationship between masticatory laterality and being underweight seems to be indirect. However, prospective studies could be designed to clarify the relationship between masticatory laterality and obesity and to show the effectiveness of eating using the more preferred chewing side.

The results of the present study also suggest that obese subjects, with not only high values of BMI but also high values of body fat percentage, eat more rapidly than subjects without obesity. This finding is in concordance with the relationship between eating fast and weight gain demonstrated in longitudinal and cross-sectional studies (Leong, Madden, Gray, Waters, \& Horwath, 2011; Tanihara et al., 2011). Our results support the idea that obese people may benefit in terms of losing weight by eating slowly. However, prospective and well controlled studies are necessary to demonstrate that faster eating predicts future weight gain, and therefore advise eating slowly in individuals who want to lose weight.

This study has several limitations. First, only one artificial test food was used to measure the first phase of food comminution; therefore, the results are applicable to only one type of food. Furthermore, because subjects are being studied, and they have to count their chewing cycles, mastication becomes a voluntary instead of a semi-automatic act. Due to the cross-sectional design of this study, we cannot demonstrate cause and effect of the relation between masticatory function and obesity.

In conclusion, being underweight and having a low body fat percentage seem to be related to a masticatory lateral asymmetry and a large cycle duration in young adults with natural dentition. In this population, masticatory performance does not seem to be related to nutritional status.

\section{Conflict of interest}

The authors confirm that there are no known conflicts of interest associated with this publication and there has been no significant financial support for this work that could have influenced its outcome.

\section{Ethical approval}

This study was approved by Secretary of research of the Autonomous University of Nayarit, Mexico. Code SIP15-20.

\section{Acknowledgements}

We are very grateful to the subjects for their kind cooperation in this study. This research received partial funding from the Complementary Support Program for Institutional Strengthening Research Groups "Repatriation" of Consejo Nacional de Ciencia y Tecnología (CONACyT) and own resources.

\section{References}

Albert, T.E., Buschang, P.H., Throckmorton, G.S., 2003 Masticatory performance: A protocol for standardized production of an artificial test food. Journal of Oral Rehabilitation 30 (7), 720-722.

Diernberger, S., Bernhardt, O., Schwahn, C., Kordass, B., 2008. Self-reported chewing side preference and its associations with occlusal, temporomandibular and prosthodontic factors: Results from the population-based Study of Health in Pomerania (SHIP-0). Journal of Oral Rehabilitation 35 (8), 613-620.

Durnin, J.V.G.A., Womersley, J.V.G.A., 1974. Body fat assessed from total body density and its estimation from skinfold thickness: Measurements on 481 men and women aged from 16 to 72 years. British Journal of Nutrition 32 (1), 77-97. 
Edlund, J., Lamm, C.J., 1980. Masticatory efficiency. Journal of Oral Rehabilitation 7 (2), 123-130.

Farias Gomes, S.G., Custodio, W., Moura Jufer, J.S., Del Bel Cury, A.A., Rodrigues Garcia, R.C.M., 2010. Correlation of mastication and masticatory movements and effect of chewing side preference. Brazilian Dental Journal 21 (4), 351-355.

Flores-Orozco, E.I., Rovira-Lastra, B., Peraire, M., Salsench, J., Martinez-Gomis, J., 2016. Reliability of a visual analog scale for determining the preferred mastication side. The Journal of Prosthetic Dentistry 115 (2), 203-208.

Flores-Orozco, E.I., Rovira-Lastra, B., Willaert, E., Peraire, M., Martinez-Gomis, J., 2016. Relationship between jaw movement and masticatory performance in adults with natural dentition. Acta Odontologica Scandinavica 74 (2), 103-107.

Flores-Orozco, E.I., 2014. Evaluación de la fiabilidad y validez de métodos que determinan el lado de preferencia masticatorio. PhD Thesis. University of Barcelona, Spain.

Gibson, R.S., 2005. Principles of nutritional assessment. Oxford University Press, USA, 273-293.

Leong, S.L., Madden, C., Gray, A., Waters, D., Horwath, C., 2011. Faster self-reported speed of eating is related to higher body mass index in a nationwide survey of middle-aged women. Journal of the American Dietetic Association $111(8), 1192-1197$.

Lujan-Climent, M., Martinez-Gomis, J., Palau, S., Ayuso-Montero, R., Salsench, J., Peraire, M., 2008. Influence of static and dynamic occlusal characteristics and muscle force on masticatory performance in dentate adults. European Journal of Oral Sciences 116 (3), 229-236.

Martín Moreno, V., Gómez Gandoy, J.B., Antoranz González, M.J., Gómez de la Cámara, A., 2003. Interchangeability among the percentages of body fat estimated by mid-arm adipose area, triceps skinfold thickness and arm-to-arm segmental bioimpedance analysis. Revista Espaíola De Salud Póblica 77 (3), 347-361.

Martinez-Gomis, J., Lujan-Climent, M., Palau, S., Bizar, J., Salsench, J., Peraire, M., 2009. Relationship between chewing side preference and handedness and lateral asymmetry of peripheral factors. Archives of Oral Biology 54 (2), 101-107.

Mizumori, T., Tsubakimoto, T., Iwasaki, M., Nakamura, T., 2003. Masticatory laterality-evaluation and influence of food texture. Journal of Oral Rehabilitation 30 (10), 995-999.

Moreno, M., Gomez Gandoy, V., Gonzales, A., 2001. Measurement of body fat with bioelectric impedance, skin fold thickness and equations based on anthropometric measurements. Revista Española De Salud Pública 75 (3), 221-236.

Okada, K., Enoki, H., Izawa, S., Iguchi, A., Kuzuya, M., 2010. Association between masticatory performance and anthropometric measurements and nutritional status in the elderly. Geriatrics \& Gerontology International 10 (1), 56-63.

Olthoff, L.W., Van Der Bilt, A., Bosman, F., Kleizen, H.H., 1984. Distribution of particle sizes in food comminuted by human mastication. Archives of Oral Biology 29 (11), 899-903.
Panel, N.O.E.I.E., 1998. Clinical guidelines on the identification, evaluation, and treatment of overweight and obesity in adults. The American Journal of Clinical Nutrition 68 (4), 899-917.

Paphangkorakit, J., Thothongkam, N., Supanont, N., 2006. Chewing-side determination of three food textures. Journal of Oral Rehabilitation 33 (1), 2-7.

Rovira-Lastra, B., Flores-Orozco, E.I., Salsench, J., Peraire, M., Martinez-Gomis, J., 2014. Is the side with the best masticatory performance selected for chewing? Archives of Oral Biology 59 (12), 1316-1320.

Rovira-Lastra, B., Flores-Orozco, E.I., Ayuso-Montero, R., Peraire, M., Martinez-Gomis, J., 2016. Peripheral, functional and postural asymmetries related to the preferred chewing side in adults with natural dentition. Journal of Oral Rehabilitation 43 (4), 279-285.

Sánchez-Ayala, A., Campanha, N.H., Garcia, R.C.M.R., 2013. Relationship between body fat and masticatory function. Journal of Prosthodontics 22 (2), 120-125.

Salsench, J., Martínez-Gomis, J., Torrent, J., Bizar, J., Samsó, J., Peraire, M., 2005. Relationship between duration of unilateral masticatory cycles and the type of lateral dental guidance: A preliminary study. International Journal of Prosthodontics 18 (4), 339-346.

Suverza, A., Haua, K.E., 2010. ABCD de la evaluación del estado de nutrición. Editorial McGraw-Hill, México DF, 15-70.

Tanihara, S., Imatoh, T., Miyazaki, M., Babazono, A., Momose, Y., Baba, M., et al., 2011. Retrospective longitudinal study on the relationship between 8-year weight change and current eating speed. Appetite 57 (1), 179-183.

Tureli, M.C.D.M., Barbosa, T.D.S., Gavião, M.B.D., 2010. Associations of masticatory performance with body and dental variables in children. Pediatric Dentistry 32 (4), 283-288.

Van der Bilt, A., Fontijn-Tekamp, F.A., 2004. Comparison of single and multiple sieve methods for the determination of masticatory performance. Archives of Oral Biology 49 (3), 193-198.

Van der Bilt, A., 2011. Assessment of mastication with implications for oral rehabilitation: A review. Journal of Oral Rehabilitation 38 (10), 754-780.

Walls and Steele,

2004. A.W.G. Walls, J.G. Steele, The relationship between oral health and nutrition in older people, Mechanisms of Ageing and Development 125 (12) (2004) 853-857.

White, A.K., Venn, B., Lu, L.W., Rush, E., Gallo, L.M., Yong, J.L.C., et al., 2015. A comparison of chewing rate between overweight and normal BMI individuals. Physiology \& Behavior 145, 8-13.

World Medical Association, 2013. World Medical Association Declaration of Helsinki: Ethical principles for medical research involving human subjects. JAMA 310 (20), 2191.

Zhu, Y., Hollis, J.H., 2015. Relationship between chewing behavior and body weight status in fully dentate healthy adults. International Journal of Food Sciences and Nutrition $66(2), 135-139$. 